Published in final edited form as:

Foot Ankle Int. 2019 April ; 40(4): 457-464. doi:10.1177/1071100718816292.

\title{
Microvasculature of the Plantar Plate Using Nano-Computed Tomography
}

\author{
Fred T. Finney, MD $^{1}$, Aaron McPheters ${ }^{2}$, Natalie V. Singer, MD $^{1}$, Jaron C. Scott, MSE ${ }^{1}$, Karl \\ J. Jepsen, PhD ${ }^{1}$, James R. Holmes, MD1, and Paul G. Talusan, MD' \\ ${ }^{1}$ Department of Orthopaedic Surgery, University of Michigan, Ann Arbor, MI, USA \\ ${ }^{2}$ University of Louisville School of Medicine, Louisville, KY, USA
}

\begin{abstract}
Background: Lesser toe plantar plate attenuation or disruption is being increasingly implicated in a variety of common clinical conditions. A multitude of surgical techniques and devices have been recently developed to facilitate surgical repair of the plantar plate. However, the microvascular anatomy, and therefore the healing potential in large part, has not been defined. We investigated the microvasculature of the plantar plate by employing a novel technique involving microvascular perfusion and nano-computed tomography (nano-CT) imaging.
\end{abstract}

Methods: Twelve human adult cadaveric lower extremities were amputated distal to the knee. The anterior and posterior tibial arteries were perfused with a barium solution. The soft tissues of each foot were then counterstained with phosphomolybdic acid (PMA). The second through fourth toe metatarsophalangeal (MTP) joints of 12 feet were imaged with nano-CT at 14-micron resolution. Images were then reconstructed for analysis of the plantar plate microvasculature and calculation of the vascular density along the length of the plantar plate.

Results: A microvascular network extends from the surrounding soft tissues at the attachments of the plantar plate on both the metatarsal and proximal phalanx. The midsubstance of the plantar plate appears to be relatively hypovascular. Analysis of the vascular density along the length of the plantar plate demonstrated a consistent trend with increased vascular density at approximately the proximal $29 \%$ and distal $22 \%$ of the plantar plate.

Conclusion: There is a vascular network extending from the surrounding soft tissues into the proximal and distal attachments of the plantar plate.

Corresponding Author: Fred T. Finney, MD, Department of Orthopaedic Surgery, University of Michigan Health System, 2098 South Main St., Ann Arbor, MI 48103, USA. ffinney@ med.umich.edu.

Authors' Note

All work was performed at the University of Michigan, Ann Arbor, Michigan.

Declaration of Conflicting Interests

The author(s) declared the following potential conflicts of interest with respect to the research, authorship, and/or publication of this article: Karl J. Jepsen, PhD, reports grants from the National Institute of Arthritis and Musculoskeletal and Skin Diseases of the National Institutes of Health, during the conduct of the study.

Paul G. Talusan, MD, reports grants from Paragon 28 and from the National Institute of Arthritis and Musculoskeletal and Skin Diseases of the National Institutes of Health, during the conduct of the study; he also reports personal fees from Paragon 28, outside the submitted work.

ICMJE forms for all authors are available online. 
Clinical relevance: The hypovascular midportion of the plantar plate may play an important role in the underlying pathoanatomy and pathophysiology of this area. These findings may have significant clinical implications for the reparative potential of this region and the surgical procedures currently described to accomplish anatomic plantar plate repair.

\section{Keywords}

plantar plate; vasculature; microvasculature; plantar plate anatomy

\section{Introduction}

Instability of the lesser toe metatarsophalangeal (MTP) joint has been implicated as the cause of some common forefoot deformities. ${ }^{32}$ The soft tissue structures surrounding the MTP joint, specifically the plantar plate and collateral ligaments, have been identified as the major stabilizing structures of this joint, with instability occurring after a tear or attenuation of these structures. ${ }^{33}$ When these static stabilizers become incompetent, an imbalance between intrinsic and extrinsic toe flexors and extensors leads to progressive, often symptomatic deformity. 5,32 These deformities include hammertoes, crossover toes, and claw toes and are known to be a major source of pain and dysfunction. ${ }^{9}$

Initial management of these deformities is typically non-operative, ranging from shoe modifications to splints to orthotic devices. However, when nonoperative treatment is unsuccessful and symptomatic MTP joint instability persists, many surgical interventions have been described. Surgical treatments are classified as nonanatomic versus anatomic repairs. Nonanatomic repairs that have been described include synovectomy, syndactylization of toes, tenotomy, tendon transfers, osteotomy, thermal shrinkage of the plantar plate, and suture tape stabilization. ${ }^{6,7,22,24,30,31,34}$ While somewhat successful in stabilizing the MTP joint, these nonanatomic repairs are reported to have residual deformity at the MTP joint in $25 \%$ to $38.5 \%$ of cases, with a similar rate of dissatisfaction postoperatively. ${ }^{14}$ As a result, there has been a trend toward anatomic repairs of the plantar plate in recent years to prevent dorsal translation of the MTP joint. ${ }^{12,38}$

Despite a body of literature emphasizing the technical aspects of direct anatomic repairs of the plantar plate, it remains unknown whether the plantar plate has the biologic capacity to heal these repairs. While the biochemical and histological character of the plantar plate and the vascular anatomy of the foot have been well studied, the vascular supply of the plantar plate has not been previously defined. ${ }^{18}$ The plantar plate has been compared to the meniscus of the knee, as both are fibrocartilaginous structures that sustain compressive loads. ${ }^{18}$ It has been well described that the meniscus is a structure with a well-vascularized periphery and central zone devoid of blood supply. ${ }^{2}$ As a result, repairs are performed in the peripheral zones but not attempted in the central zone where the watershed blood supply limits the ability to heal these repairs. Defining and quantifying the vascular supply of the plantar plane may provide further insight into the pathophysiology of plantar plate disruption as well as provide a better understanding of the potential for healing after surgical repairs.

Nano-computed tomography (nano-CT) has been widely used in the analysis of vascularity and bony microarchitecture of animals and other human anatomical structures. ${ }^{10,19,21,37}$ 
However, the utility of nano-CT technology in analyzing soft tissue structures has been comparatively limited, but it holds great promise for acquiring 3D images of complex anatomical structures like the plantar plate. ${ }^{20}$ In this study, we sought to define the microvascular anatomy of the plantar plate using nano-CT imaging.

\section{Methods}

This study utilized de-identified human cadaver specimens, defined by our institutional review board as unregulated human tissue use, and was determined exempt from review. Thirteen fresh-frozen human cadaveric lower-extremity specimens, amputated below the knee, were utilized in this study. They were kept frozen at $-20^{\circ} \mathrm{C}$ and were allowed to thaw at room temperature for 24 hours prior to use. All specimens were between the ages of 18 and 65 years and had no history of diabetes, peripheral vascular disease, or connective tissue disorders. Additionally, specimens were excluded if they had history of trauma to the foot or ankle, prior foot or ankle surgery, or evidence of clinical deformity of the lesser toes upon inspection.

\section{Specimen Perfusion and Counterstaining}

The anterior and posterior tibial arteries were carefully dissected and transected above the ankle for cannulation with a 16-gauge blunt-tip needle. The proximal ends of the transected arteries were clamped to prevent leakage via collateral vessels. Each artery was then flushed under constant pressure with 5\% PI 3N1 Pre-Injection Embalming Fluid (Trinity Fluids, Inc., Lapeer, MI) in distilled water until venous return was free of blood clots. One hundred twenty milliliters of a $15 \%$ barium sulfate suspension in $10 \%$ neutral buffered formalin (Thermo Fischer, Kalamazoo, MI), a radio-opaque contrast, was then injected into each artery. As each artery was injected, the other artery, not in use, was clamped to prevent leakage. Specimens were then refrigerated for 24 hours and amputated at the proximal metatarsals for imaging.

The x-ray attenuation of soft tissues was enhanced using phosphomolybdic acid (PMA) (Sigma-Aldrich, Louis, MO). Because pilot testing demonstrated that the skin impaired PMA penetration into the deep soft tissues, the skin was removed prior to counterstaining. Specimens were then soaked in a 3-L bath of $10 \mathrm{~g} / \mathrm{L}$ PMA solution for a minimum of 9 days. Each bath was kept on a shaker table to ensure agitation, and the PMA solutions were changed every 3 days.

\section{Imaging and Analysis}

The MTP joints of the second, third, and fourth toes were scanned in a GE Nanotom S nanoCT scanner (GE Measurements, Wunstorf, Germany) at a resolution of $14 \mu \mathrm{m}$. Images were viewed and analyzed with Dragonfly (v3.1, Object Research Systems, Inc., Montreal, QC, Canada). The region of the plantar plate was manually identified and selected for analysis. The barium sulfate perfused vasculature was identified as a separate region using image thresholding. The vascular density, defined as vessel volume over tissue volume (VV/TV), was calculated along the longitudinal axis of the plantar plate. Because the plantar plate is a complex, curvilinear, 3D structure and software analysis was limited to a linear analysis, the 
longitudinal axis of the plantar plate was estimated. To do this, the midpoints between the plantar and dorsal aspects of the proximal and distal attachments were identified, and linear analysis was carried out along this axis. The percentage of vascular density was then calculated along the percentage length of the plantar plate, on this axis, for standardization between different specimen lengths.

\section{Histology}

Histological analysis was performed on 2 second toe specimens to validate imaging results. Two specimens were perfused with black India ink, $100 \mathrm{~mL}$ per artery, after injection with preembalming fluid as described above. The specimens were again flushed with preembalming fluid. Specimen perfusion, sectioning, counterstaining, and imaging were then carried out as described above. After imaging, the second toe MTP joint of each specimen was removed en bloc and plastic embedded in poly-methyl methacrylate for histological sectioning. Each joint was then sectioned in the sagittal plane using a thin sectioning pathology saw (Exakt Technologies, Inc., Oklahoma City, OK) to obtain 300- $\mu$ m slices. A Nikon Eclipse Ni-U microscope was used for analysis, and images were captured with a DS-Fi2 digital camera. NIS BR software (Nikon Instruments, Inc., Melville, NY) was used for image processing. Qualitative comparisons were made between nano-CT imaging and thin sectioned slices.

\section{Results}

A total of 35 lesser toes from 10 human cadaveric specimens were included in the final study analysis. One cadaveric foot specimen was excluded due to clinical deformities of the second and third toes and a poor-quality scan of the fourth toe. One additional fourth toe sample was excluded due to poor scan quality with motion artifact. The final study group included 12 second toe samples, 12 third toe samples, and 11 fourth toe samples. There were an equal number of right and left feet from 6 male and 4 female cadaveric specimens, with an average age of 50.3 years. The average length of the plantar plate was $19.9 \mathrm{~mm}$, as measured on nano-CT imaging. In observation of both the nano-CT imaging and the histological evaluation, the plantar plate of the lesser toes was noted to have microvascular networks at the proximal and distal attachments, while the midsubstance of the plantar plate appeared relatively hypovascular.

\section{Nano-CT Imaging Evaluation}

In gross evaluation of imaging, all plantar plate specimens demonstrated similar microvascular infiltration at the proximal attachments to the metatarsal neck and plantar fascia, as well as at the distal attachment on the proximal phalanx (Figure 1). Proximally, the plantar plate received a vascular supply from the surrounding soft tissues, including the interosseous muscles, and the periosteum of the metatarsal neck. No single vessel could be identified as the primary contributor to this vascular network. Moving distally along the plantar plate, the microvasculature became notably less dense and appeared nearly avascular in much of the midsubstance. At the distal attachment, the microvascular density increased, again provided by the surrounding soft tissues. Similar to the proximal attachment, no single vessel was identified as a primary contributor. However, the vasculature projected into the 
MTP joint at the central recess dorsally between the inferior articular border of the proximal phalanx and the point of attachment of the plantar plate. ${ }^{28}$

Quantitative analysis of the plantar plate microvasculature confirmed the qualitative observations made of the imaging. Vascular densities, as percentage vessel volume over total tissue volume of the plantar plate, are shown along the longitudinal axis of the plantar plate (Figure 2). While there was variation between specimens, all toe specimens demonstrated increased vascular densities at the proximal and distal aspects. The average vascular densities for each toe type and overall average vascular density for all toe samples are shown in Figure 3. On average, a hypovascular region, which we defined as having a vascular density less than half of the maximum vascular density, existed in the region between $30 \%$ and $78 \%$ from the proximal attachment to the distal attachment along the length of the plantar plate when all 35 specimens were grouped together. Descriptive findings of the quantitative analysis for each toe type are presented in Table 1.

\section{Histological Evaluation}

Two second toe specimens were used to qualitatively verify nano-CT imaging results. Histological analysis demonstrated microvascular networks infiltrating the proximal and distal plantar plate attachments, consistent with the regions observed and identified on analysis of nano-CT imaging. The microvasculature was similarly noted to dissipate toward the midsubstance of the plantar plate, with much of the midsubstance again appearing nearly avascular (Figure 4).

\section{Discussion}

Defining the vascular anatomy of the plantar plate is important for developing a better understanding of the pathophysiology and pathoanatomy of plantar plate tears and lesser toe deformities. Additionally, as anatomic surgical repairs of plantar plate tears are increasing in popularity, ${ }^{38}$ it is important to gain a better understanding of the healing potential of this soft tissue structure where the vascular anatomy has not been previously described. In this study, we implemented a novel technique using nano-CT imaging to analyze the microvasculature of the plantar plate. Our results demonstrate that the plantar plate receives its vascular supply almost exclusively at the proximal and distal attachments. This vascular supply is provided by the surrounding soft tissues and extends into approximately the proximal 20\% to $30 \%$ and distal $20 \%$ of the plantar plate along the longitudinal axis. At the distal plantar plate attachment, the vasculature projects into the synovial recess between the articular cartilage of the proximal phalanx and the plantar plate attachment. The remaining midsubstance region of the plantar plate appears relatively hypovascular by comparison.

The gross and microscopic anatomy as well as the histochemistry of the normal plantar plate has been well described. ${ }^{8,11,15,18,36}$ This anatomic structure is a trapezoidal ligament that consists of fibrocartilage and primarily longitudinally oriented type I collagen and acts as the primary stabilizer of the MTP joint in the sagittal plane. ${ }^{8,11,23,33}$ Proximally, the plantar plate attaches to the distal aspect of the plantar fascia as well as to the metatarsal neck periosteum. The plantar plate attaches distally on the plantar base of the proximal phalanx, and there is a central recess at the dorsal aspect of the plantar plate between the inferior 
articular border of the proximal phalanx and the point of attachment. ${ }^{8,11,28}$ However, little description of the vasculature is provided by these studies. In a histological anatomic study by Gregg et al, vessels are noted to "appear to enter peripherally, mainly plantarly," but no further description is provided. ${ }^{15}$ However, they did note vascularized tissue projecting into the distal joint recess, which was consistently identified in the present study. In another study, which aimed to correlate sonographic findings of cadaveric plantar plates with MRI and histology, the normal plantar plate was noted to have "a relatively small number of blood vessels." 17

It has been well established that a tissue's potential for healing is directly linked to the arterial blood supply available to deliver an inflammatory response and nutritional support necessary for healing. ${ }^{3}$ Knowledge of vascular anatomy is fundamental in understanding pathophysiology and therefore surgical repair techniques and indications. For example, a hypovascular area has been consistently identified in the midsubstance of the Achilles tendon, and this area has been associated with increased risk of rupture and surgical complications. ${ }^{4,27}$ In the foot, fractures of the proximal fifth metatarsal and tarsal navicular are notorious for being more difficult to heal due to the watershed blood supply in these areas. ${ }^{25,26,35,39}$ The knee meniscus was identified to have vascularity existing only at the peripheral third, which has established guidelines for reparable and irreparable tears. ${ }^{2,41}$ In general, ligaments represent relatively hypovascular tissues, and different ligaments demonstrate different potentials for revascularization during healing. ${ }^{1,3}$ The present study identified increased vascularity at the proximal and distal attachments of the plantar plate with a relatively hypovascular midsubstance. However, no definitive conclusions may be drawn from these data regarding the plantar plate's potential for revascularization during the healing response.

Dissatisfaction rates following anatomic plantar plate repair for treatment of lesser MTP joint instability remain relatively high, and restoring anatomic alignment, allowing the affected toe to rest on the ground during static stance, is often not achieved or maintained. $13,16,29,40$ Present clinical studies are limited to short- to medium-term results. ${ }^{13,16,29,40}$ Long-term follow-up of clinical and radiographic evaluations and/or patient-reported outcomes is unreported. Additionally, further investigation is needed to better understand how the hypovascular midsubstance of the plantar plate may relate to clinical pathology, surgical indications, and healing potential. Nery et al[AQ: 1] presented a series of 40 plantar plate repairs in 22 patients in which all MTP joints first underwent arthroscopic grading of tears. However, there was little description of tear location along the length of the plantar plate. A better understanding of tear location, specifically as it relates to the vascularity and relative healing potential of the region, may provide better indications for anatomic repairs and improved outcomes.

Limitations of this study include the use of a cadaveric model and the challenges associated with validating a $3 \mathrm{D}$ imaging technique. Factors that may have limited our ability to visualize small blood vessels include the use of frozen cadaveric tissue, which may have disrupted some of the microvasculature; the degree to which our methods allowed for adequate microvascular perfusion; and the resolution of the nano-CT images, which were reconstructed at 14 microns. Though our outcomes were qualitatively confirmed on 
histological evaluation, it is possible that imaging did not provide a completely accurate quantitative analysis. Moreover, the definition of "hypovascular" based on vascular density was determined by the authors for a comparative analysis, as a numerical value for vascular density as related to healing potential has not been previously established.

Future work by the current authors will focus on plantar plate pathology and tear location as related to vascular zones. A similar analysis of lesser toe MTP joints with clinical deformity may not only provide further insight into how vascular anatomy may relate to pathologic conditions but also help us to understand the plantar plate's potential for revascularization and healing. Though excluded from final analysis in the present study, 2 lesser MTP joints with clinical deformity were imaged using the previously described protocol. Each plantar plate had an identifiable tear on imaging and demonstrated an unexpected increase in vascularity at the site of the tear. Similarly, Gregg et al noted on their histological evaluation of abnormal plantar plates that the average number of blood vessels more than doubled. ${ }^{17}$ Our small sample of pathologic plantar plates suggests that tears may incite a neovascular response, but further investigation is needed.

\section{Conclusion}

In conclusion, our analysis showed a vascular network extending from the surrounding soft tissues into approximately the proximal $20 \%$ to $30 \%$ and the distal $20 \%$ of the plantar plate. The hypovascular midportion of the plantar plate may play an important role in the underlying pathoanatomy and pathophysiology of this area. This is the first study to describe the 3D vascular anatomy of the plantar plate, developing and employing an effective, novel technique for soft tissue imaging and vascular analysis.

\section{Acknowledgments}

Funding

The author(s) disclosed receipt of the following financial support for the research, authorship, and/or publication of this article: U.S. Department of Health and Human Services.[AQ: 2] Funding for this study was provided by Paragon 28. Research reported in this publication was supported by the National Institute of Arthritis and Musculoskeletal and Skin Diseases of the National Institutes of Health under award number P30 AR069620. The content is solely the responsibility of the authors and does not necessarily represent the official views of the National Institutes of Health.

\section{References}

1. Arnoczky SP, Rubin RM, Marshall JL. Microvasculature of the cruciate ligaments and its response to injury. An experimental study in dogs. J Bone Joint Surg Am 1979;61(8): 1221-1229. [PubMed: 511882]

2. Arnoczky SP, Warren RF. Microvasculature of the human meniscus. Am J Sports Med. 1982;10(2): 90-95. [PubMed: 7081532]

3. Bray RC, Rangayyan RM, Frank CB. Normal and healing ligament vascularity: a quantitative histological assessment in the adult rabbit medial collateral ligament. J Anat. 1996;188(pt 1):87-95. [PubMed: 8655419]

4. Chen TM, Rozen WM, Pan W-R, Ashton MW, Richardson MD, Taylor GI. The arterial anatomy of the Achilles tendon: anatomical study and clinical implications. Clin Anat. 2009;22(3):377-385. [PubMed: 19173244]

5. Coughlin MJ. Crossover second toe deformity. Foot Ankle. 1987;8(1):29-39. [PubMed: 3623359] 
6. Coughlin MJ. Second metatarsophalangeal joint instability in the athlete. Foot Ankle. 1993;14(6): 309-319. [PubMed: 8406245]

7. Daly PJ, Johnson KA. Treatment of painful subluxation or dislocation at the second and third metatarsophalangeal joints by partial proximal phalanx excision and subtotal webbing. Clin Orthop Relat Res. 1992;(278):164-170.

8. Deland JT, Lee KT, Sobel M, DiCarlo EF. Anatomy of the plantar plate and its attachments in the lesser metatarsal phalangeal joint. Foot Ankle Int 1995;16(8):480-486. [PubMed: 8520660]

9. Doty JF, Coughlin MJ. Metatarsophalangeal joint instability of the lesser toes and plantar plate deficiency. J Am Acad Orthop Surg. 2014;22(4):235-245. [PubMed: 24668353]

10. Duvall CL, Taylor WR, Weiss D, Guldberg RE. Quantitative microcomputed tomography analysis of collateral vessel development after ischemic injury. Am J Physiol Heart Circ Physiol. 2004;287(1):H302-H310. [PubMed: 15016633]

11. Finney FT, Cata E, Holmes JR, Talusan PG. Anatomy and physiology of the lesser metatarsophalangeal joints. Foot Ankle Clin. 2018;23(1):1-7. [PubMed: 29362026]

12. Finney FT, Lee S, Scott J, Irwin TA, Holmes JR, Talusan PG. Biomechanical evaluation of suture configurations in lesser toe plantar plate repairs. Foot Ankle Int. 2018;39(7):836-842. [PubMed: 29460645]

13. Flint WW, Macias DM, Jastifer JR, Doty JF, Hirose CB, Coughlin MJ. Plantar plate repair for lesser metatarsophalangeal joint instability. Foot Ankle Int. 2017;38(3):234-242. [PubMed: 27852647]

14. Gazdag A, Cracchiolo A. Surgical treatment of patients with painful instability of the second metatarsophalangeal joint. Foot Ankle Int. 1998;19(3):137-143. [PubMed: 9542983]

15. Gregg J, Marks P, Silberstein M, Schneider T, Kerr J. Histologic anatomy of the lesser metatarsophalangeal joint plantar plate. Surg Radiol Anat. 2007;29(2):141-147. [PubMed: 17318282]

16. Gregg J, Silberstein M, Clark C, Schneider T. Plantar plate repair and Weil osteotomy for metatarsophalangeal joint instability. Foot Ankle Surg. 2007;13(3):116-121.

17. Gregg JM, Silberstein M, Schneider T, Kerr JB, Marks P. Sonography of plantar plates in cadavers: correlation with MRI and histology. AJR Am J Roentgenol. 2006;186(4):948-955. [PubMed: 16554562]

18. Johnston RB, Smith J, Daniels T. The plantar plate of the lesser toes: an anatomical study in human cadavers. Foot Ankle Int. 1994;15(5):276-282. [PubMed: 7951967]

19. Kadar A, Morsy M, Sur Y-J, Laungani AT, Akdag O, Moran SL. The vascular anatomy of the capitate: new discoveries using micro-computed tomography imaging. J Hand Surg. 2017;42(2): $78-86$.

20. Khoury BM, Bigelow EMR, Smith LM, et al. The use of nano-computed tomography to enhance musculoskeletal research. Connect Tissue Res. 2015;56(2):106-119. [PubMed: 25646568]

21. Kline TL, Zamir M, Ritman EL. Relating function to branching geometry: a micro-CT study of the hepatic artery, portal vein, and biliary tree. Cells Tissues Organs 2011;194(5): 431-442. [PubMed: 21494011]

22. Klinge SA, McClure P, Fellars T, DiGiovanni CW. Modification of the Weil/Maceira metatarsal osteotomy for coronal plane malalignment during crossover toe correction: case series. Foot Ankle Int 2014;35(6):584-591. [PubMed: 24651712]

23. Maas NMG, van der Grinten M, Bramer WM, Kleinrensink G-J. Metatarsophalangeal joint stability: a systematic review on the plantar plate of the lesser toes. J Foot Ankle Res. 2016;9:32. [PubMed: 27547243]

24. Mann RA, Mizel MS. Monarticular nontraumatic synovitis of the metatarsophalangeal joint: a new diagnosis? Foot Ankle. 1985;6(1):18-21. [PubMed: 4043887]

25. McKeon KE, Johnson JE, McCormick JJ, Klein SE. The intraosseous and extraosseous vascular supply of the fifth metatarsal: implications for fifth metatarsal osteotomy. Foot Ankle Int. 2013;34(1):117-123. [PubMed: 23386771]

26. McKeon KE, McCormick JJ, Johnson JE, Klein SE. Intraosseous and extraosseous arterial anatomy of the adult navicular. Foot Ankle Int. 2012;33(10):857-861. [PubMed: 23050710] 
27. Munteanu SAchilles tendon. In: Rome K, McNair P, eds. Management of Chronic Conditions in the Foot and Lower Leg. London, UK: Churchill Livingstone; 2015:145-179.

28. Nery C, Coughlin MJ, Baumfeld D, et al. Lesser metatarsal phalangeal joint arthroscopy: anatomic description and comparative dissection. Arthroscopy 2014;30(8):971-979. [PubMed: 24835840]

29. Nery C, Coughlin MJ, Baumfeld D, Mann TS. Lesser metatarsophalangeal joint instability: prospective evaluation and repair of plantar plate and capsular insufficiency. Foot Ankle Int. 2012;33(4):301-311. [PubMed: 22735202]

30. Nery C, Coughlin MJ, Baumfeld D, Raduan FC, Mann TS, Catena F. Prospective evaluation of protocol for surgical treatment of lesser MTP joint plantar plate tears. Foot Ankle Int. 2014;35(9): 876-885. [PubMed: 24958766]

31. Nery C, Raduan FC, Catena F, Mann TS, de Andrade MAP, Baumfeld D. Plantar plate radiofrequency and Weil osteotomy for subtle metatarsophalangeal joint instablity. J Orthop Surg Res. 2015;10:180. [PubMed: 26584658]

32. Shirzad K, Kiesau CD, DeOrio JK, Parekh SG. Lesser toe deformities. J Am Acad Orthop Surg. 2011;19(8):505-514. [PubMed: 21807918]

33. Suero EM, Meyers KN, Bohne WHO. Stability of the metatarsophalangeal joint of the lesser toes: a cadaveric study. J Orthop Res. 2012;30(12):1995-1998. [PubMed: 22696467]

34. Sung W Technique using interference fixation repair for plantar plate ligament disruption of lesser metatarsophalangeal joints. J Foot Ankle Surg. 2015;54(3):508-512. [PubMed: 24973038]

35. Torg JS, Pavlov H, Cooley LH, et al. Stress fractures of the tarsal navicular. A retrospective review of twenty-one cases. J Bone Joint Surg Am. 1982;64(5):700-712. [PubMed: 7085695]

36. Umans H, Srinivasan R, Elsinger E, Wilde GE. MRI of lesser metatarsophalangeal joint plantar plate tears and associated adjacent interspace lesions. Skeletal Radiol. 2014;43(10):1361-1368. [PubMed: 24880715]

37. van Alphen NA, Morsy M, Laungani AT, et al. A three-dimensional micro-computed tomographic study of the intraosseous lunate vasculature: implications for surgical Intervention and the Development of Avascular Necrosis. Plast Reconstr Surg. 2016;138(5):869e-878e.

38. Watson TS, Reid DY, Frerichs TL. Dorsal approach for plantar plate repair with Weil osteotomy: operative technique. Foot Ankle Int. 2014;35(7):730-739. [PubMed: 24850163]

39. Waugh W The ossification and vascularisation of the tarsal navicular and their relation to Köhler's disease. J Bone Joint Surg Br. 1958;40-B(4):765-777. [PubMed: 13610991]

40. Weil L, Sung W, Weil LS, Malinoski K. Anatomic plantar plate repair using the Weil metatarsal osteotomy approach. Foot Ankle Spec. 2011;4(3):145-150. [PubMed: 21421939]

41. Yagishita K, Muneta T, Ogiuchi T, Sekiya I, Shinomiya K. Healing potential of meniscal tears without repair in knees with anterior cruciate ligament reconstruction. Am J Sports Med. 2004;32(8):1953-1961. [PubMed: 15572327] 

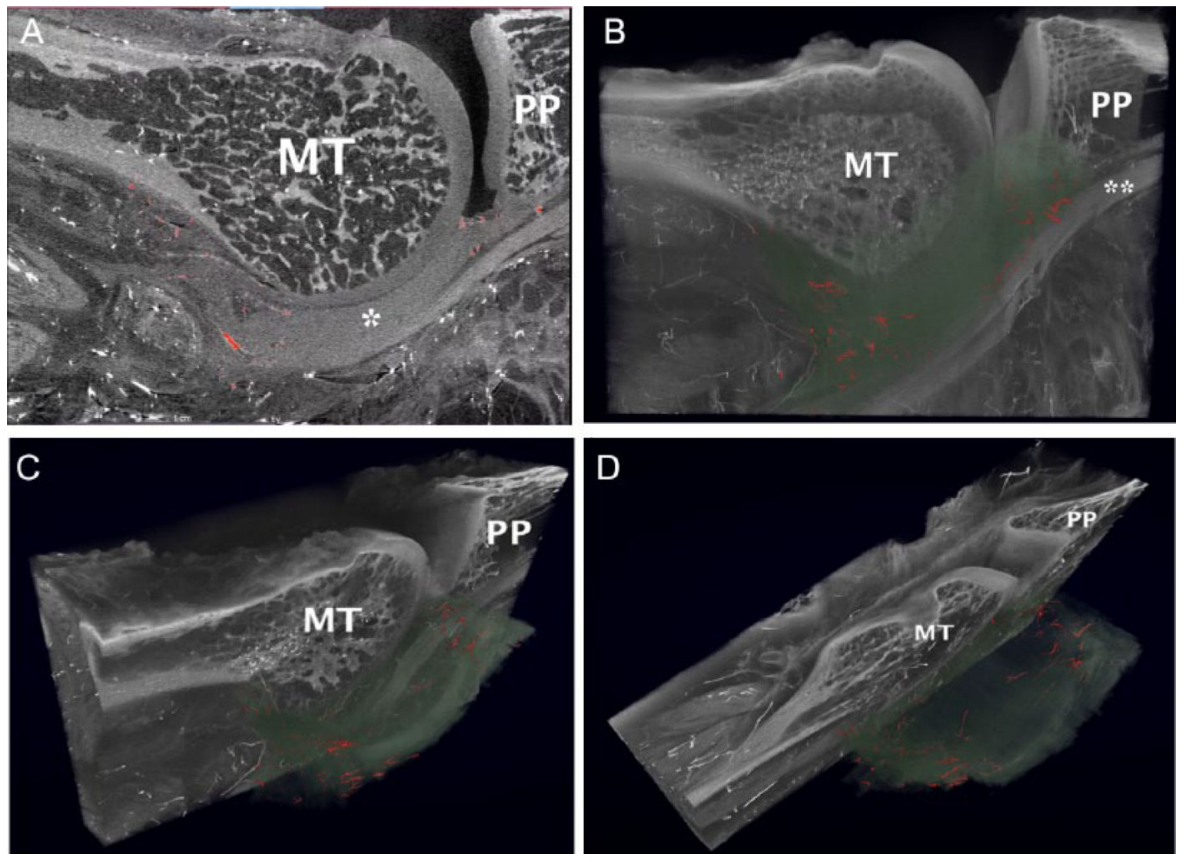

Figure 1.

(A) 2D sagittal nano-CT slice and (B-D) 3D reconstruction of lesser toe MTP joint nano-CT scan. (A) Representative sagittal cut on 2D analysis. The barium-infused vasculature appears as bright white in the nano-CT images. The vasculature within the plantar plate (*) was pseudocolored red for visualization purposes. (B) Sagittal view of a 3D reconstruction of a lesser toe MTP joint with lateral bone and soft tissues surrounding the plantar plate removed. The plantar plate is highlighted in green and semitranslucent with the vasculature colored red. The metatarsal head (MT), proximal phalanx (PP), and flexor tendon (**) are labeled for reference. (C and D) Oblique sagittal and dorsal views, respectively, of the 3D reconstructed lesser toe MTP joint with lateral bone and soft tissues surrounding the plantar plate removed. Vascularized tissue is noted to project into the distal recess between the articular cartilage of the proximal phalanx and the distal plantar plate attachment. 


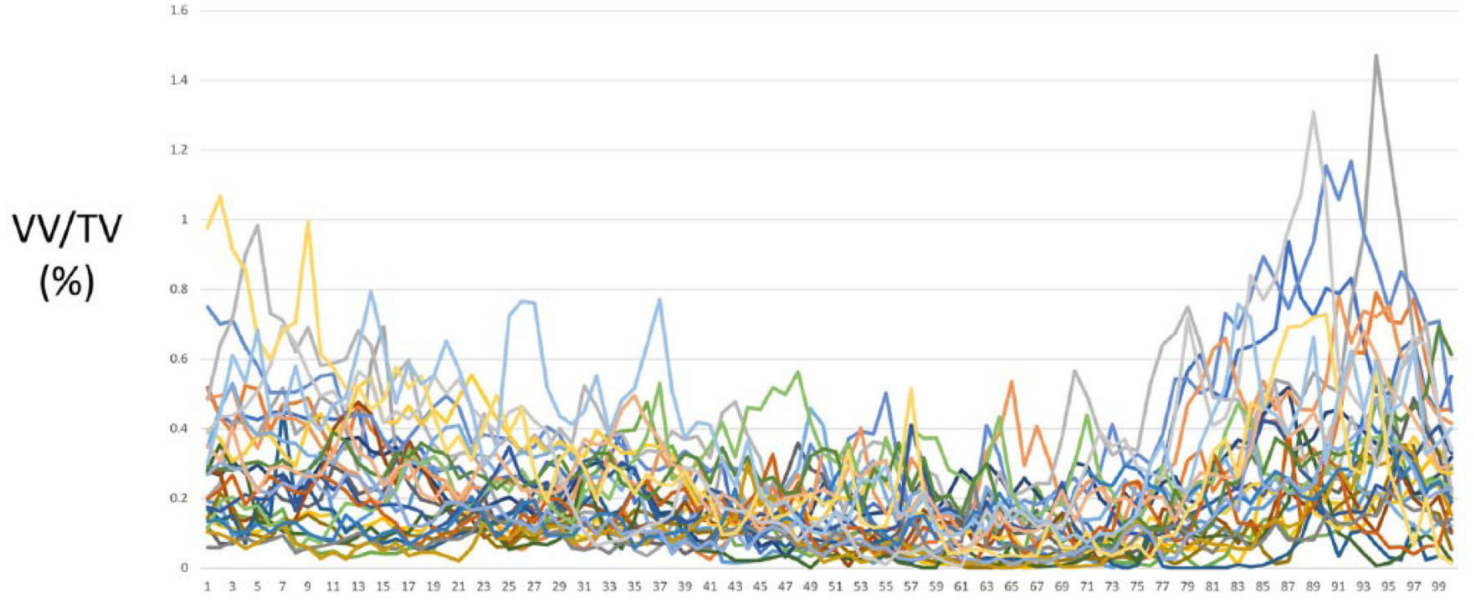

Percentage Length Along the Plantar Plate from Proximal to Distal

Figure 2.

Vascular densities of all specimens. Vascular density (as percent vessel volume per tissue volume [VV/TV] of the plantar plate) was calculated along the percentage length of each plantar plate specimen from the proximal attachment $(0 \%)$ to the distal attachment $(100 \%)$. While there was variation between specimens, all plantar plate specimens demonstrated increased vascular densities at the proximal and distal aspects. 
VV/TV

(\%)

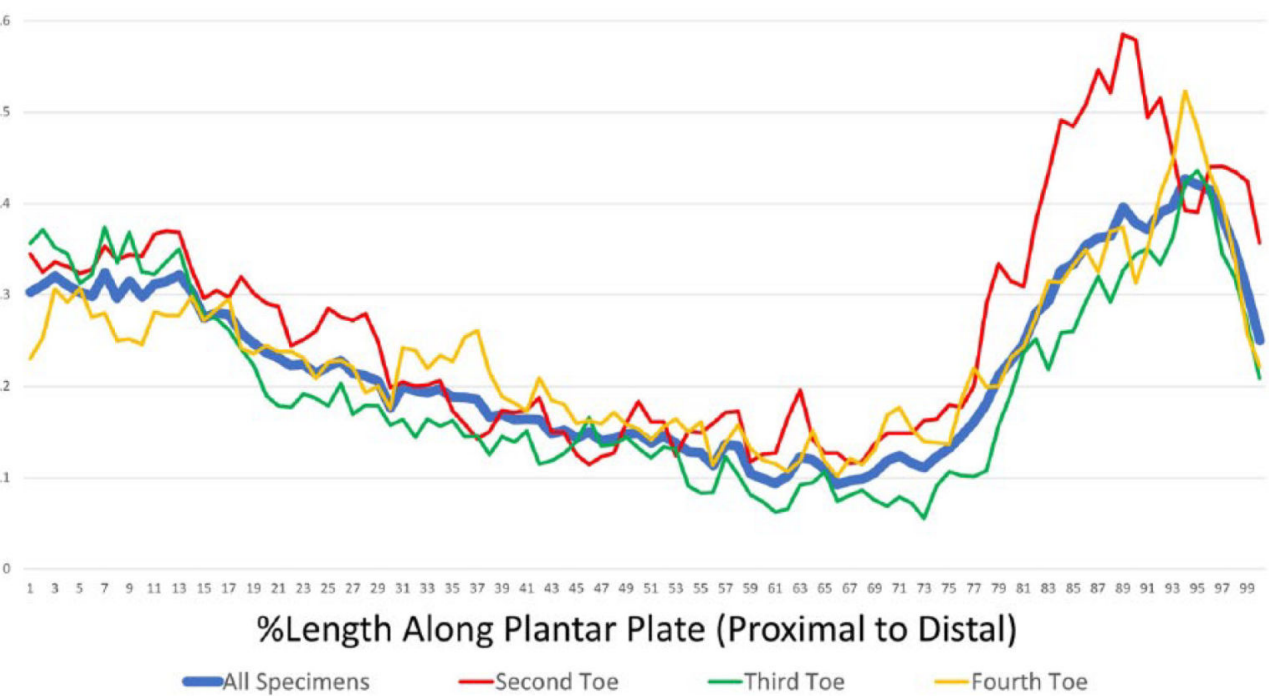

Figure 3.

Average vascular density. Average vascular density (as percent vessel volume per tissue volume [VV/TV] of the plantar plate) was calculated along the percentage length of each plantar plate by toe type from the proximal attachment (0\%) to the distal attachment (100\%). A consistent trend was noted across all toe specimens demonstrating increased vascular densities at the proximal and distal aspects. 


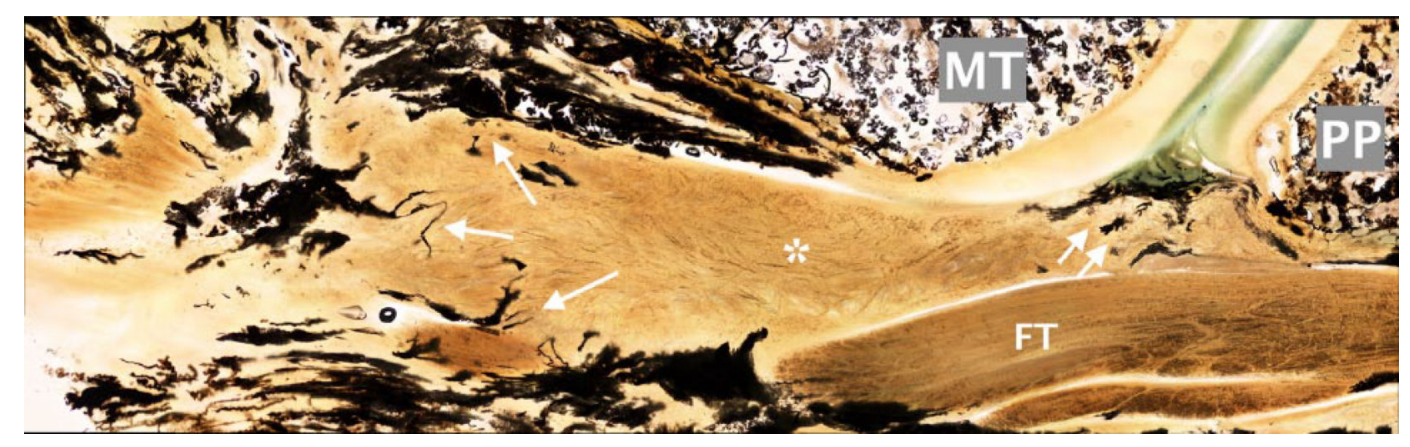

Figure 4.

Histological evaluation of the plantar plate vasculature. Midsagittal section of plantar aspect of a second toe metatarsophalangeal joint at $4 \times$ magnification with the proximal aspect to the left and the distal aspect to the right. The metatarsal head (MT; top), proximal phalanx (PP; top right), and flexor tendons (FT) are shown for reference. The plantar plate (*) demonstrates increased vascularity at the proximal (single arrows) and distal (double arrows) aspects. Vascularized tissue is noted to project into the distal recess between the articular cartilage of the proximal phalanx and the distal plantar plate attachment. 


\section{롤 \\ 골}

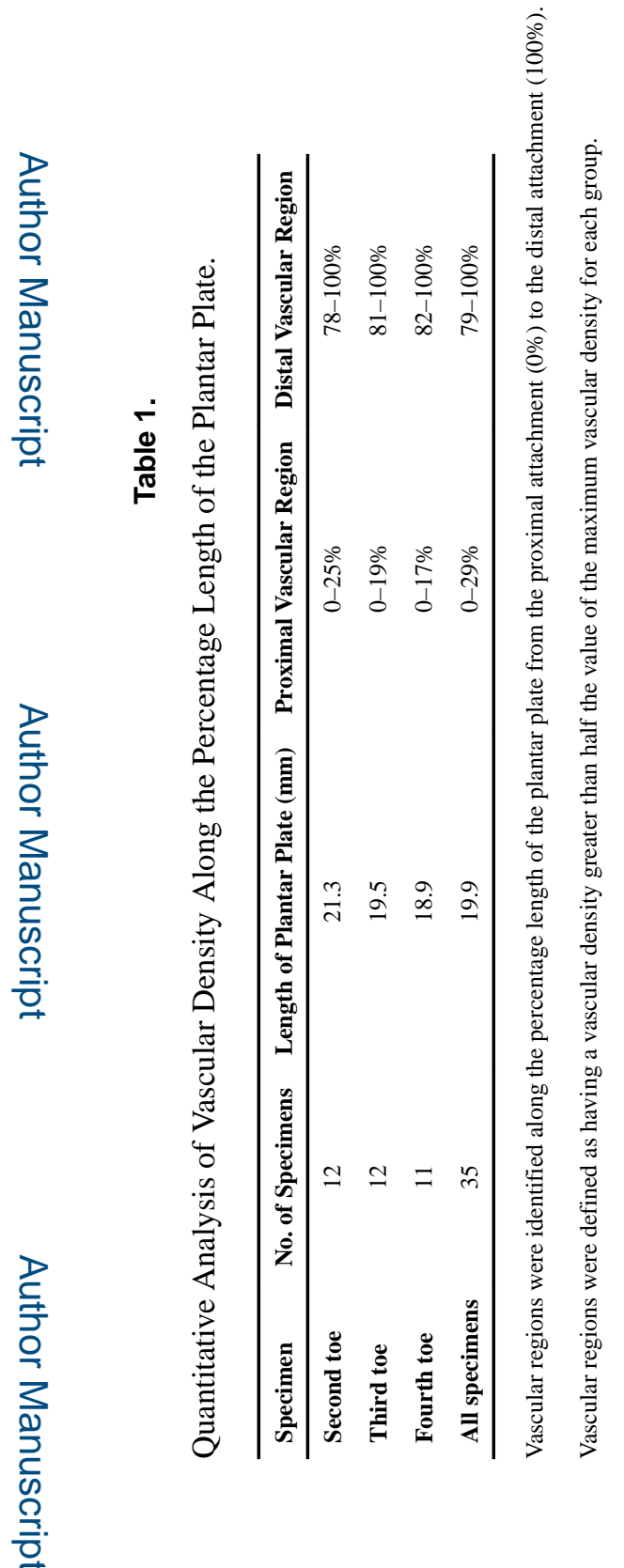

Foot Ankle Int. Author manuscript; available in PMC 2020 April 01. 